\title{
PATH OPTIMIZATION OF COLD CHAIN DISTRIBUTION WITH MULTIPLE DISTRIBUTION CENTERS CONSIDERING CARBON EMISSIONS
}

\author{
REN, X. Y. ${ }^{1}-$ CHEN, C. F. ${ }^{1}-$ XIAO, Y. L. ${ }^{1,2^{*}}-$ DU, S. C. ${ }^{1}$ \\ ${ }^{I}$ School of Management Engineering and Business, Hebei University of Engineering, Handan \\ 056038, China \\ ${ }^{2}$ Faculty of Business, Economics and Accountancy, University Malaysia Sabah, Kota Kinabalu \\ 88400, Malaysia \\ *Corresponding author \\ e-mail: 12357414@qq.com
}

(Received $3^{\text {rd }}$ Apr 2019; accepted $17^{\text {th }}$ May 2019)

\begin{abstract}
With the growing awareness of green logistics, the carbon emissions must be considered in the cold chain distribution (CCD). Whereas the traditional regional distribution mode cannot deliver multiple small batches to designated regions right on time, this paper establishes a mathematical model for the minimal distribution cost of fresh food based on the resource/information sharing between multiple distribution centers (multi-DC) and the soft time window constraint, aiming to satisfy the new demands on logistics distribution (e.g. high timeliness, low cost, greenness and resource/information sharing). The total cost refers to the sum of vehicle dispatch cost, transport cost, time penalty cost, cargo loss cost and carbon emissions cost. Next, the proposed model was solved by the hybrid algorithm of artificial fishswarm algorithm (AFSA) and ACA (AFSA-ACA hybrid algorithm). The simulation examples show that the multi-DC CCD mode can effectively reduce the total distribution cost and carbon emission cost compared to the traditional regional CCD mode.
\end{abstract}

Keywords: green logistic, low carbo distribution, semi-open cold chain distribution (CCD), artificial fish-swarm algorithm (AFSA), ant colony algorithm (ACA)

\section{Introduction}

Facing the growing demand for fresh food, cold chain distribution (CCD) enterprises are constantly increasing the number of vehicles and distribution centers (DCs), aiming to deliver multiple small batches to designated regions right on time. The semi-open multi-DC joint CCD fully uses the existing vehicles and lowers the distribution cost. This distribution mode overcomes the defects of the traditional regional distribution mode, such as insufficient distribution capacity, the lack of resources, the inability to share information, and the regional limitation. Therefore, it is very important to explore the path optimization of semi-open multi-DC CCD.

In recent years, fruitful results have been achieved on CCD path optimization, which is a type of vehicle routing problem (VRP) (Dantzig and Ramser, 1959). For example, Lan et al. (2013) classified CCD modes, helped enterprises choose the suitable collaborative distribution model, and reduced the cost of enterprise distribution. For the minimal distribution cost, Wang et al. (2018) established and solved an optimization model for multi-temperature cold-storage CCD under stochastic demand.

Considering the limitation of the standard algorithms, some scholars have introduced improved algorithms or the time window constraint to explore the CCD path optimization. For instance, Wang and Luo (2017) optimized the CCD path with time window using the improved intelligent water drops algorithm. To coordinate the time 
window of consumer demand with the temperature control of fresh food, Wang et al. (2019) constructed an optimization model that minimizes the distribution cost and value loss of fresh food, and solved the model using a hybrid algorithm between the genetic algorithm (GA) and the tabu search (TS) (hybrid GA-TS). Liang et al. (2016) included the fuzzy time window into the CCD, set up a multi-objective optimization model for the minimal cost and maximum consumer satisfaction, and solved the model by the improved GA.

With the boom of third-party logistics, the traditional regional single-DC distribution mode can no longer fulfil the demand of distribution enterprises, and is being replaced by the open and semi-open multi-DC distribution modes. On open multi-DC distribution, Yang et al. (2016), Yu and Xie (2011) and Baldacci et al. (2013) all assumed that any vehicle must leave from a virtual DC to the actual DCs, before implementing the distribution tasks, and the trip between the virtual and actual DCs incurs no cost. Considering the diversity and mutual exclusion of products, the various types of vehicles and the matching between products and vehicles, Liu and Guo (2016) put forward the open-loop multi-DC path optimization problem with time window constraint, set up the corresponding mathematical model, and solved the model with hybrid GA and the adaptive GA, respectively. Ge et al. (2016) proposed the multi-DC distribution mechanism with open vehicle parking strategy, and created and solved a mathematical model for the minimal fuel consumption. Liu et al. (2010) employed the two-stage heuristic algorithm to solve the multi-yard distribution VRP under the constraint of vehicle load.

On semi-open multi-DC CCD, Fan et al. (2018) presented a semi-open multi-DC distribution mode, constructed a mathematical model for the minimum cost under time window constraint, and solved the fresh food CCD by the ant colony algorithm (ACA). Ho et al. (2007), Adelzadeh et al. (2014) and Liu (2013) respectively solved the multiyard VRP under time window constraint with the hybrid GA, simulated annealing (SA) algorithm and the adaptive GA based on artificial bee colony (ABC) algorithm.

The carbon emissions should be considered in the path optimization of CCD (Chaabane et al., 2012), as required by the government's policies on carbon emissions reduction, the concept of green logistics development, and the coordination between CCD and ecological environment. Gac (1996) examined the relationship between CCD development and greenhouse effect, and put forward measures to curb the CCD impacts on greenhouse effect. Vanek and Sun (2008) investigated the relationship between the maintenance of food shelf life, energy consumption and environmental pollution, built a relevant energy consumption model, and proposed the strategy for selection of transport modes. James et al. (2010) studied how climate change and temperature rise influence food quality, and designed measures to improve the energy efficiency in each link of the CCD. Considering carbon emissions, Kang et al. (2019) and Fan et al. (2017) both constructed CCD path optimization models for the minimal total cost under time window constraint, and solved the simulation examples by the ACA improved by the 2opt local search mechanism and the improved GA, respectively. Palmer (2007) established a path optimization model with time window, with the aim to optimize distance, time and carbon emissions, and verified the $\mathrm{CO}_{2}$ emissions reduction effect of speed through the analysis on the variation in $\mathrm{CO}_{2}$ emissions under different congestion conditions. Bao and Zhang (2018) introduced the carbon emissions into the path optimization model of semi-open multi-DC CCD, and solved the model by the improved GA. 
The research results on $\mathrm{CCD}$ both bring economic benefits to enterprises and promote the greenness of the CCD. However, the existing studies on semi-open multiDC CCD fail to fully consider the cost incurred in the distribution process, or the insufficiency of self-owned vehicles (some vehicles are rented from third parties). To overcome the defects of these studies, this paper designs a global network covering all consumers, assuming that the enterprise has multiple DCs and sufficient vehicles, and establishes a comprehensive semi-open multi-DC CCD model constrained by soft time window. The model attempts to minimize the sum of vehicle dispatch cost, transport cost, time penalty cost, cargo loss cost and carbon emissions cost. In addition, the proposed model was solved by the hybrid algorithm of artificial fish-swarm algorithm (AFSA) and ACA (AFSA-ACA hybrid algorithm). The simulation examples show that the multi-DC CCD mode is better than the traditional regional CCD mode.

\section{Materials and methods}

\section{Problem description}

This paper mainly studies the "many-to-many" semi-open multi-DC CCD mode. In this cross-regional network distribution mechanism, "many" DCs serve "many" consumers, and share with each other the consumer information, resources and vehicles. Considering carbon emissions, a semi-open multi-DC CCD path optimization model was established to deliver multiple small batches to designated regions. Below is a description of our research problem.

It is assumed that a distribution enterprise serves $\mathrm{N}$ consumers with $\mathrm{M}$ DCs and $\mathrm{K}$ vehicles. During the distribution, each DC should be open to all vehicles, and play the role of the final destination or stopover point. After completing the distribution task, a vehicle does not need to return to the original DC, but to any of the nearby DCs. The consumers only receive distribution services and do not allow the stopover of vehicles. The vehicles are sufficient to satisfy the distribution demand. However, the route, distance and stopover DCs vary with vehicles and trips, due to the limitations of the load, speed and distance of the vehicles. The distance between each DC and each consumer is known, so is the distance between consumers. This paper designs a penalty function based on time window, because late distribution will damage the quality of fresh food and cause dissatisfaction among consumers. The carbon emissions from the CCD are mainly from energy consumption and refrigeration.

Based on the above, this paper constructs a mathematical model to minimize the total cost, which covers vehicle dispatch cost, transport cost, time penalty cost, cargo loss cost and carbon emissions cost. Besides, the improved GA was designed to solve the model and optimize the delivery path. The optimal path can minimize the path length, increase vehicle utilization, lower carbon emissions and reduce the total cost of the distribution service. Figure 1 illustrates the semi-open multi-DC CCD mode.

\section{Model hypotheses}

(1) The vehicles are of the same model and the same specifications. The maximum load, fuel consumption and refrigeration performance are known in advance.

(2) There are enough vehicles to satisfy the demand of all consumers.

(3) The order quantity completed by each vehicle should not surpass the maximum load. 
(4) The demand of each consumer can only be fulfilled by one vehicle through one service.

(5) Each consumer sets out a time window on the delivery time, and will not accept the service before the earliest acceptable time or after the latest acceptable time.

(6) The position of each DC and each consumer is known in advance. Every DC boasts sufficient cargoes and the ability to serve the consumer demand.

(7) All vehicles must start from a DC. After completing the distribution task, a vehicle does not need to return to the original DC, but to any of the nearby DCs.

(8) Two drivers are arranged for each vehicle, so that they can take turns to rest. The labor cost and vehicle operation cost are both included in the dispatch cost.

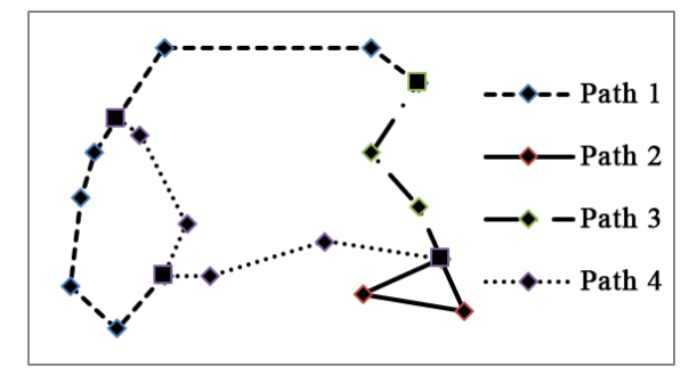

Figure 1. Sketch map of the semi-open multi-DC CCD mode

\section{Model construction}

Symbols

$M$ : The set of DCs, $M\{m|m=1,2, \ldots| M \mid\}$;

$N$ : The set of consumers, $N\{n|n=1,2, \ldots| N \mid\}$;

$K$ : The set of vehicles, $K\{k|k=1,2, \ldots| K \mid\}$;

$K_{n 1}$ : The set of consumers served by vehicle $k$ on the $l$-th distribution trip, $N_{k 1} \in N, 0 \leq 1 \leq$ num, num $=|N|$, where num is the number of elements in $N$;

$Q:$ The maximum load of each vehicle;

$q_{j}$ : The demand of consumer $j, q_{j}>0$;

$d_{i j}$ : The distance between consumer $i$ and consumer $j$;

$i$ and $j$ : The serial number of nodes, $\forall i, j \in M \cup N$;

$X_{i j}^{k}$ : The vehicle movement variable; if vehicle $k$ moves from consumer $i$ to consumer $j$, then $X_{i j}^{k}=1$, otherwise, $X_{i j}^{k}=0$;

$S_{k}$ : The time that vehicle $k$ leaves from DC for the last trip;

$v$ : The vehicle speed;

$C_{1}$ : The dispatch cost per trip of a vehicle;

$C_{2}$ : The transport cost per unit distance of a vehicle;

$C_{3}$ : The unit time penalty cost for early arrival or late delivery of a vehicle;

$C_{4}$ : The cargo loss cost of a vehicle;

$P$ : The unit value of fresh food;

$\theta_{1}$ : The proportion of cargo loss en route;

$\theta_{2}$ : The proportion of cargo loss in loading/unloading;

$T_{j}^{k}$ : The time vehicle $k$ arrives at consumer $j$; 
$t_{i}$ : The time vehicle $k$ serves at consumer $i$;

$C_{0}$ : The unit carbon emissions cost.

\section{Mathematical model}

(1) Time penalty cost. To ensure the temperature and humidity of fresh food and satisfy the consumer's time requirement, the distributor must deliver the cargoes within the agreed time period. However, the cargoes may not be delivered within the agreed period for various reasons in actual distribution. Hence, the concept of soft time window emerged: the distributor can deliver the cargoes beyond the agreed time period but within the maximum tolerable period of the consumer, while bearing a certain time penalty cost (Dai and Liang, 2017). In this paper, a penalty function is designed according to the time window of the consumers. Specifically, no penalty cost will be incurred if the cargoes are delivered within the period $\left[E_{j}, L_{j}\right]$ agreed by consumer $j$ and the distributor; the consumer will not accept any service beyond the period $\left[E T_{j}, L T_{j}\right]$, where $E T_{j}$ and $L T_{j}$ are respectively the earliest and latest delivery times the consumer can tolerate; a certain penalty cost will be incurred according to the arrival time of the vehicle if the cargoes are delivered between $\left[E T_{j}, E_{j}\right]$ and $\left[L_{j}, L T_{j}\right]$. The time windows of consumer $j$ are described as Figure 2.

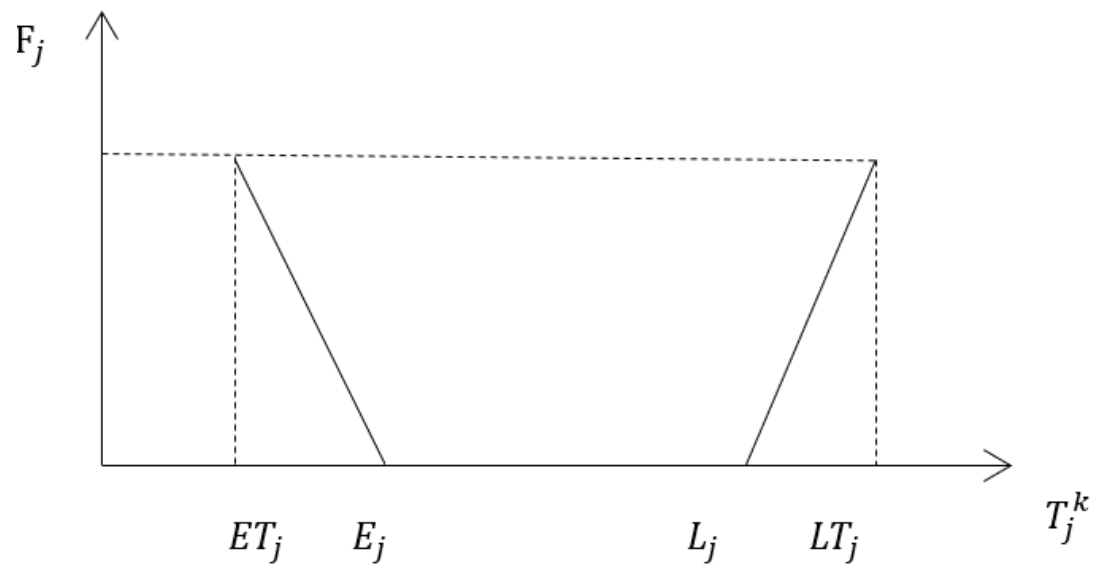

Figure 2. Sketch map of the time windows of consumer $j$

The time penalty cost can be expressed as Equation 1:

$$
F_{j}\left(T_{j}^{k}\right)=\left\{\begin{array}{ll}
C_{3}\left(E_{j}-T_{j}^{k}\right), & E T_{j} \leq T_{j}^{k} \leq E_{j} \\
0, & E_{j} \leq T_{j}^{k} \leq L_{j} \\
C_{3}\left(T_{j}^{k}-L_{j}\right), & L_{j} \leq T_{j}^{k} \leq L T_{j}
\end{array} ;\right.
$$

(2) Cargo loss cost. The CCD of fresh food must meet the requirements on temperature and humidity. The fresh food may suffer from a certain loss during the long-distance transport and loading/unloading (Fan et al., 2017). Considering the effects of transport and loading/unloading on the quality and value of fresh food, this paper assumes that the temperature remains constant in the vehicle during distribution, and 
excludes the impacts from other factors. In other words, the cargo loss of fresh food is assumed to be correlated with the distribution distance and the door opening in loading/unloading. Hence, the cargo loss cost was calculated based on the two issues. Let $P\left(\theta_{1} d_{i j}+\theta_{2} q_{j}\right)$ be the cargo loss cost incurred as vehicle $j$ moves from consumer $i$ to consumer $j$ and completes the delivery to consumer $j$. Then, the total cargo loss cost through the distribution process can be calculated as Equation 2:

$$
C_{4}=P \sum_{i \in M \cup N} \sum_{j \in K \cup N} \sum_{k \in K} x_{i j}^{k}\left(\theta_{1} d_{i j}+\theta_{2} q_{j}\right)
$$

(3) Carbon emissions cost. The CCD releases more $\mathrm{CO}_{2}$ than the distribution of ordinary cargoes. The carbon emissions in the CCD mainly comes from fuel combustion, which depends on the transport distance is and the cargo load (Xiao et al., 2012; Duro, 2013). The fuel consumption per unit distance $\mu$ can be regarded as a linear function of the cargo load $q: \mu(q)=a\left(Q_{0}+q\right)+b$ where $Q_{0}$ is the dead load of the vehicle, and $a$ and $b$ are linear function coefficients. When a vehicle is under fully load, the fuel consumption per unit distance $\mu^{*}=a\left(Q_{0}+Q\right)+b$; when a vehicle is under zero load, the fuel consumption per unit distance $\mu_{0}=a Q_{0}+b$. To sum up, the fuel consumption can be expressed as: $\mu(q)=\mu_{0}+\left(\frac{\left(\mu^{*}-\mu_{0}\right)}{Q} q\right)$. Let $Q_{i j}$ be the load of vehicle $k$ moving from consumer $i$ to consumer $j$, and $\mu\left(Q_{i j}\right)$ be the fuel consumption of vehicle $k$ per unit of distance. Then, the carbon emissions cost can be expressed as $C\left(Q_{i j}\right)=C_{0} d_{i j} e_{0} \mu\left(Q_{i j}\right)$.

Based on the above description and hypotheses, the path optimization model for the minimal total distribution cost can be established as Equation 3:

$$
\begin{aligned}
& \operatorname{MinC}=C_{1} \sum_{i \in M \cup N} \sum_{j \in M \cup N} \sum_{k \in K} x_{i j}^{k}+C_{2} \sum_{i \in M \cup N} \sum_{j \in M \cup N} \sum_{k \cup K} x_{i j}^{k} d_{i j} \\
& +\sum_{j \in M \cup N} \sum_{k \cup K} F_{j}\left(T_{j}^{k}\right)+P \sum_{i \in M \cup N} \sum_{j \in M \cup N} \sum_{k \cup K} x_{i j}^{k}\left(\theta_{1} d_{i j}+\theta_{2} q_{j}\right) \\
& +C_{0} e_{0} \sum_{i \in M \cup N} \sum_{j \in M \cup N} \sum_{k \in K} d_{i j} \mu\left(Q_{i j}\right) x_{i j}^{k}
\end{aligned}
$$

s.t.

$$
\begin{gathered}
\sum_{j \in N} X_{i j}^{k} q_{j} \leq Q, \forall i \in M \\
\sum_{i \in M \cup N} \sum_{k \in K} X_{i j}^{k}=1, \forall j \in N \\
\sum_{j \in M \cup N} \sum_{k \in K} X_{i j}^{k}=1, \forall i \in N
\end{gathered}
$$




$$
\begin{gathered}
\sum_{i \in M} \sum_{j \in M} x_{i j}^{k}=0, \forall k \in K \\
\sum_{i \in M} \sum_{j \in N} x_{i j}^{k}=\sum_{i \in N} \sum_{j \in M} x_{i j}^{k}, \forall k \in K \\
\sum_{i \in N_{k l} j \in N_{k l}} X_{i j}^{k} \leq\left|N_{k l}\right|-1, \forall k \in K, \forall N_{k l} \subseteq N, 0 \leq 1 \leq n u m \\
T_{j}^{k}=\sum_{i \in M \cup N}\left(T_{i}^{k}+\frac{d_{i j}}{V}+t_{i}\right) X_{i j}^{k}, k \in K, j \in M \cup N \\
X_{i j}^{k}=\{0,1\}, \forall i, j \in M \cup N, \forall k \in K
\end{gathered}
$$

where Equation 3 is the objective function about the minimal total cost of distribution, including the vehicle dispatch cost, transport cost, time penalty cost, cargo loss cost and carbon emissions cost; Equations 4-12 are the constraints of the model, which respectively specifies the vehicle load cannot surpass the maximum load, that each consumer can only be served once, that a vehicle must leave the consumer after completing the task, that a vehicle cannot move from a DC directly to another DC, that a vehicle can return to any DC after completing the task, that the branch constraint is removed, that each vehicle must deliver cargoes in strict accordance with the sequence of consumers, that the time vehicle $k$ arrives at customer $j$, and that $x_{i j}^{k}=1$ if vehicle $k$ moves from consumer $i$ to consumer $j$ ( $x_{i j}^{k}=0$ if otherwise).

\section{Algorithm design}

Assuming the existence of a virtual DC, this paper attempts to solve the semi-open multi-DC CCD path optimization problem as the AFSA-ACA hybrid algorithm. The hybrid algorithm was proposed by introducing the crowding factor of the AFSA to the ACA. This factor can adjust the pheromone concentration left by the ants on the search paths, and thus control the aggregation behavior of the ant colony, leading to the optimal solution. In this way, the AFSA-ACA hybrid algorithm manages to avoid the local optimal trap. The original ACA often falls into this trap due to the following reasons: In the ACA, the path selection of the ant colony is easily affected by the pheromone concentration. Thus, the suboptimal solution often appears in advance, and the pheromones continue to accumulate on the suboptimal paths.

\section{$A C A$}

The ACA was first proposed by the Italian scholar Dorigo and Gambardella (1997). For an ant $k$ in path selection, the probability that the ant moves from node $i$ to node $j$ at time $t$ can be calculated from the pheromone concentration and heuristic information on the path between the two nodes, as shown in Equation 13: 


$$
P_{i j}^{k}(t)= \begin{cases}{\left[\tau_{i j}\right]^{\alpha}\left[\eta_{i j}\right]^{\beta} / \sum_{j \in J_{k}(i)}\left[\tau_{i j}\right]^{\alpha}\left[\eta_{i j}\right]^{\beta},} & j \in J_{k}(i) \\ 0, & \text { otherwise }\end{cases}
$$

where: $\tau_{i j}$ is the residual pheromone concentration on the path between node $i$ and node $j ; \eta_{i j}=1 / d_{i j}$ is a heuristic function about the expectation of ant $k$ to move from node $i$ to node $j$ (the smaller $d_{i j}$, the greater $\eta_{i j}$ and the higher $P_{i j}^{k}(t)$; $J_{k}(i)=\{1,2, \ldots, n\}-t a b u_{k}$ is the set of cities that ant $k$ is allowed to choose from, with tabu $_{k}$ being the tabu list of ant $k$ (the nodes that the ant has served); $\alpha$ is the relative importance of pheromone concentration; $\beta$ is the importance of the heuristic function, i.e. the importance the ant attaches to the heuristic information.

After one cycle, the global pheromone of the distribution optimization plan involving all ants should be updated. Following the ant-cycle update strategy, the pheromone concentration on each path can be updated by Equations 14-16:

$$
\begin{gathered}
\tau_{i j}(t+n)=(1-\rho) \tau_{i j}(t)+\Delta \tau_{i j} \\
\Delta \tau_{i j}=\sum_{k=1}^{K} \Delta \tau_{i j}^{k} \\
\Delta \tau_{i j}^{k}= \begin{cases}\frac{Z}{L_{k}}, & \text { if ant } k \text { passes through path } e(i, j) \text { in the current cycle; } \\
0, & \text { if otherwise. }\end{cases}
\end{gathered}
$$

where $\rho$ is the pheromone evaporation rate $0<\rho \leq 1 ; 1-\rho$ is the persistence coefficient of the pheromone); $\Delta \tau_{i j}^{k}$ is the pheromone increment on the path $e(i, j)$ in this iteration; $\Delta \tau_{i j}^{k}$ is the amount of pheromone released by ant $k$ on the path $e(i, j)$ in this iteration; $Z$ is a constant; $L_{k}$ is the path length covered by ant $k$ in this iteration; $e(i, j)$ is the path from node $i$ to node $j$.

\section{$A F S A$}

Proposed by Li et al. (2002), the AFSA divides the behaviors of fish swarm into foraging, clustering, tailgating and random behaviors by mimicking the activites of actual fish. During foraging and tailgating, the fish swarm will cluster in the area rich in food, which is similar to the search for the optimal solution. To prevent premature convergence, the crowding factor $\delta$ is introduced to control the aggregation concentration of the fish swarm, thus avoiding the local optimal trap.

Let $\delta(t)$ be the crowding factor at time $t, X_{i}$ be the current state of an artificial fish (AF) and $Y_{i}$ be the food concentration. Then, the AF searches for the number $n_{f}$ of its partners and the center position $X_{c}$ of the current neighborhood. During clustering, 
if $Y_{c} / n_{f}>\delta(t) Y_{i}$, then the fish swarm will move one step toward the center position of the partners; otherwise, the fish swarm will start foraging.

During tailgating, if $Y_{j} / n_{f}>\delta Y_{i}$, then the fish swarm will move one step toward the direction of the partner with the best state $X_{j}$ in the search space; otherwise, the fish swarm will start foraging. Here, $X_{i}, n_{f}$ and $Y_{j}$ have the same meanings as above.

\section{AFSA-ACA hybrid algorithm}

In our AFSA-ACA hybrid algorithm, the crowding factor $\delta\left(N_{c}\right)$ in the $N c$-th iteration can be expressed as Equation 17:

$$
\delta\left(N_{c}\right)=\gamma e^{-c n}
$$

where $y$ is the closeness to the extreme value; $c$ is the coefficient of variation.

Following the ant-cycle update rule, the crowding factor $\varepsilon_{i j}\left(N_{c}\right)$ of the phenomenon on the transfer target path in the $N c$-th iteration acquired by the ACA can be expressed as Equations 18:

$$
\varepsilon_{i j}\left(N_{c}\right)=1-\tau_{i j}\left(N_{c}\right) / \sum_{i \neq j} \tau_{i j}
$$

In each iteration, our AFSA-ACA hybrid algorithm first uses the ACA and the roulette strategy to compute the transfer probability of ants between the consumers that satisfy all constraints conditions (e.g. time window and maximum load) and exist outside the tabu list, to select the next consumer to be served, and determine the transfer target path. Then, the AFSA is called to compute the pheromone crowdedness on that path, to see if the path is selectable. If $\varepsilon_{i j}\left(N_{c}\right)>\delta\left(N_{c}\right)$, the path will be selected; otherwise, the ant will stopover at a nearby actual DC, remove the vehicle information and finally return to the virtual DC. If the ant can go on with distribution, it should replenish the cargoes at the actual DC it visited before returning to the virtual DC. Assuming that no cost is incurred and no time is consumed in the replenishment, the actual DC will be added to the current solution. Then, the transfer probabilities will be recalculated and the consumer will be selected again.

The specific flow of the AFSA-ACA hybrid algorithm is illustrated in Figure 3.

\section{Simulation and discussion}

\section{Calculation example}

There is no universal set of examples for semi-open multi-DC CCD, for the distribution mode is subjected to multiple constraints. This paper carries out simulation using the data (Fan et al., 2018). The calculation example is shown in Table 1.

It is assumed that a fresh food logistics enterprise W owns one virtual DC and 4 actual DCs, and serves 48 main consumers. The enterprise operates sufficient refrigerated vehicles, which have the same specifications. The maximum load $Q$ is $10 \mathrm{t}$, the mean speed is $60 \mathrm{~km} / \mathrm{h}$, the vehicle dispatch $\operatorname{cost} C_{1}$ is RMB 600 yuan, and the 
transport cost per unit of distance $C_{2}$ is RMB 10 yuan. The enterprise and each consumer agree to deliver the fresh food within the time window $\left[E_{j}, L_{j}\right]$, where $E_{j}$ is between 6 and 9 and $L_{j}$ is between 9 and 17. The earliest tolerable delivery time $E T_{j}$ is $2 \mathrm{~h}$ before the $E_{j}$, and the latest tolerable delivery time $L T_{j}$ is $2 \mathrm{~h}$ after the $L_{j}$. The time penalty cost $C_{3}$ is RMB 50 yuan/h if the fresh food is delivered earlier than $E_{j}$ or later than $L_{j}$. The value of the fresh food is RMB 5,000 yuan/t. The proportion of cargo loss en route $\theta_{1}$ is $0.1 \%$, and that in loading/unloading $\theta_{2}$ is $0.2 \%$. The $\mathrm{CO}_{2}$ emissions coefficient $e_{0}$ is $2.61 \mathrm{~kg} / \mathrm{L}$, and the unit cost of carbon emissions $C_{0}$ is $\mathrm{RMB}$ 0.1 yuan $/ \mathrm{kg}$. For a fully-loaded vehicle, the fuel consumption per unit distance $\mu_{0}$ is $1 \mathrm{~L} / \mathrm{km}$; for a zero-loaded vehicle, the fuel consumption per unit distance $\mu^{*}$ is $2 \mathrm{~L} / \mathrm{km}$. The paths between different nodes are considered as straight lines. The distance between consumers and that between consumer and DC are computed by $d_{i j}=\sqrt{\left(x_{i}-x_{j}\right)^{2}+\left(y_{i}-y_{j}\right)^{2}}$.

\section{Distribution modes}

Inspired by the decomposition method, the traditional distribution mode firstly sorts the 48 consumers through k-means clustering, and allocates them to 4 DCs. Then, the distribution plan was prepared to minimize the total distribution cost. In this plan, each $\mathrm{DC}$ is responsible for the distribution to the consumers in the specified region; each vehicle must return to the original DC after completing the tasks in the responsible region.

The proposed distribution mode considers the 48 consumers as a whole and makes uniform planning of the distribution plan. In this mode, each vehicle can stopover or replenish its cargoes after serving a batch of consumers, until completing all distribution tasks.

In this section, both distribution modes are solved by the AFSA-ACA hybrid algorithm. Through repeated simulations on the example, the basic parameters of the hybrid algorithm were set as: $N_{c \max }=1000$, Ant num $_{1}=50, \alpha=1, \beta=3$, and $\rho=0.5$. The $\tau_{i j}$ was initialized as 1 . The crowding factors were computed as $\gamma=0.9$ and $c=0.0002$. The simulations were run on Matlab2014b and the optimal paths of the two distribution modes were subjected to comparative analysis.

Figure 4 shows the optimal path of enterprise M obtained for the traditional mode. A total of 7 vehicles are needed to serve the 48 consumers, which were allocated to the 4 DCs by k-means clustering. The consumers and paths of each vehicle are listed in Table 2.

Figure 5 shows the optimal path of enterprise $\mathrm{M}$ obtained for the proposed mode. In this mode, the 48 consumers are viewed as a whole and the 4 DCs share resources with each other. After completing the tasks of the original DC, each vehicle can stopover or replenish its cargoes at a nearby DC; then, the vehicle can continue with distribution until completing all consumer orders in the whole network. A total of 5 vehicles are needed to serve all consumers in this mode. The consumers and paths of each vehicle are listed in Table 3. 


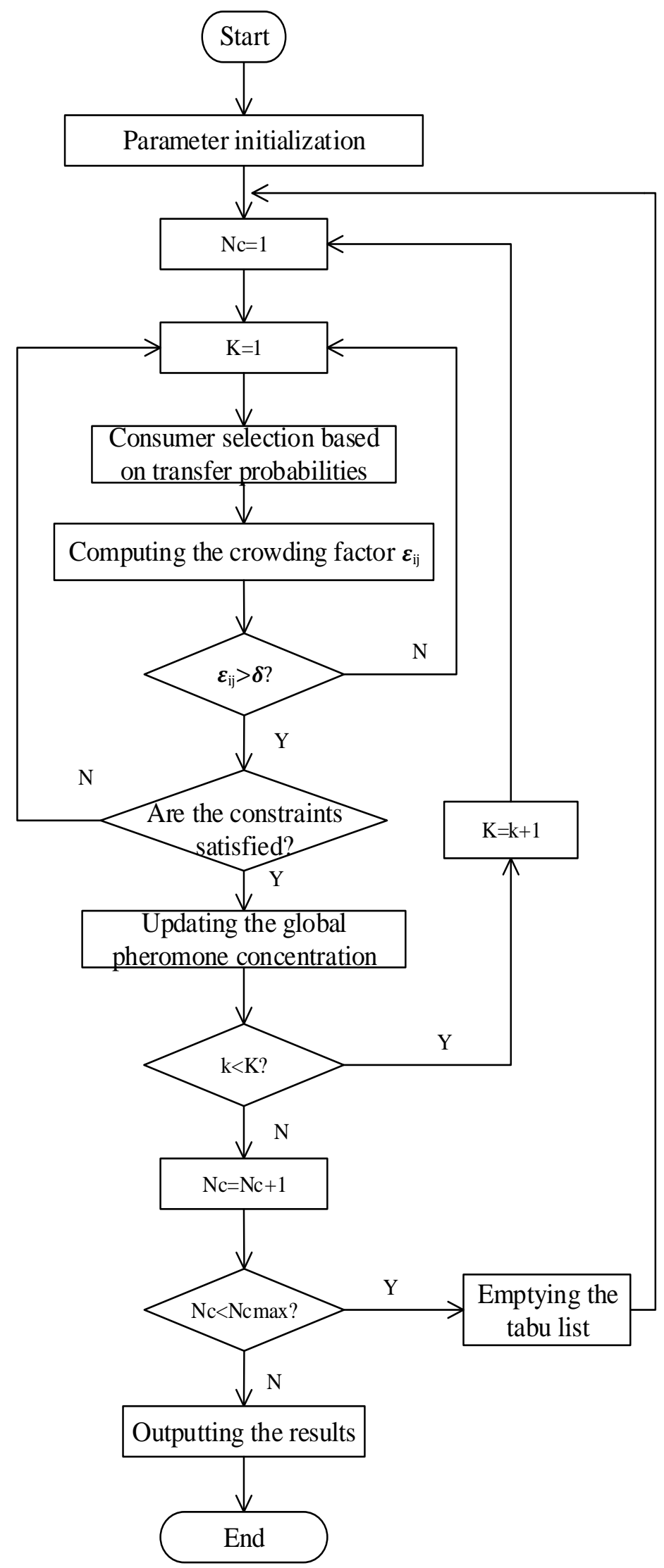

Figure 3. The workflow of the AFSA-ACA hybrid algorithm 
Table 1. Information on the multi-DC calculation example

\begin{tabular}{|c|c|c|c|c|c|c|c|c|c|}
\hline \multirow[b]{2}{*}{ No. } & \multirow[b]{2}{*}{ Node } & \multicolumn{2}{|c|}{ Position coordinates } & \multirow{2}{*}{ Requirement } & \multirow[b]{2}{*}{ Operation time } & \multicolumn{4}{|c|}{ Time windows } \\
\hline & & $X$ & $y$ & & & $E T_{j}$ & $E_{j}$ & $L_{j}$ & $L T_{j}$ \\
\hline 1 & Virtual Distribution Center & - & - & - & - & - & - & - & - \\
\hline 2 & Distribution Center A & 4.16 & 13.56 & 0 & 0 & & 6 & 19 & \\
\hline 3 & Distribution Center B & 21.39 & 17.11 & 0 & 0 & & 6 & 19 & \\
\hline 4 & Distribution Center C & -36.12 & 49.10 & 0 & 0 & & 6 & 19 & \\
\hline 5 & Distribution Center D & -31.20 & 0.24 & 0 & 0 & & 6 & 19 & \\
\hline 6 & Customer Point 1 & -29.73 & 64.14 & 1.2 & 0.40 & 4.5 & 6.5 & 10.0 & 12.0 \\
\hline 7 & Customer Point 2 & -30.66 & 5.46 & 0.8 & 0.27 & 5.5 & 7.5 & 16.5 & 18.5 \\
\hline 8 & Customer Point 3 & 51.64 & 5.47 & 1.6 & 0.53 & 6.5 & 8.5 & 15.5 & 17.5 \\
\hline 9 & Customer Point 4 & -13.17 & 69.34 & 0.5 & 0.17 & 5.5 & 7.5 & 16.5 & 18.5 \\
\hline 10 & Customer Point 5 & -67.41 & 68.32 & 1.2 & 0.40 & 6.5 & 8.5 & 13.5 & 15.5 \\
\hline 11 & Customer Point 6 & 48.91 & 6.27 & 0.5 & 0.17 & 5.5 & 7.5 & 16.5 & 18.5 \\
\hline 12 & Customer Point 7 & 5.24 & 22.26 & 1.3 & 0.43 & 4.5 & 6.5 & 9.5 & 11.5 \\
\hline 13 & Customer Point 8 & -65.00 & 77.23 & 2.0 & 0.67 & 6.5 & 8.5 & 13.5 & 15.5 \\
\hline 14 & Customer Point 9 & -4.18 & -1.57 & 1.3 & 0.43 & 4.5 & 6.5 & 10.0 & 12.0 \\
\hline 15 & Customer Point 10 & 23.03 & 11.64 & 1.8 & 0.60 & 5.5 & 7.5 & 16.5 & 18.5 \\
\hline 16 & Customer Point 11 & 25.48 & 6.29 & 0.7 & 0.23 & 6.5 & 8.5 & 13.5 & 15.5 \\
\hline 17 & Customer Point 12 & -42.62 & -26.39 & 0.6 & 0.20 & 5.5 & 7.5 & 16.5 & 18.5 \\
\hline 18 & Customer Point 13 & -76.67 & 99.34 & 0.9 & 0.30 & 5.5 & 7.5 & 15.5 & 17.5 \\
\hline 19 & Customer Point 14 & -20.67 & 57.89 & 0.9 & 0.30 & 4.5 & 6.5 & 10.5 & 12.5 \\
\hline 20 & Customer Point 15 & -52.04 & 6.57 & 0.4 & 0.13 & 4.5 & 6.5 & 9.5 & 11.5 \\
\hline 21 & Customer Point 16 & -41.38 & 50.82 & 2.5 & 0.83 & 5.5 & 7.5 & 16.5 & 18.5 \\
\hline 22 & Customer Point 17 & -91.94 & 27.59 & 0.5 & 0.17 & 5.5 & 7.5 & 15.5 & 17.5 \\
\hline 23 & Customer Point 18 & -65.12 & 30.21 & 1.7 & 0.57 & 4.5 & 6.5 & 9.5 & 11.5 \\
\hline 24 & Customer Point 19 & 18.60 & 96.72 & 0.3 & 0.10 & 5.5 & 7.5 & 16.5 & 18.5 \\
\hline 25 & Customer Point 20 & -40.94 & 83.21 & 1.6 & 0.53 & 4.5 & 6.5 & 12.0 & 14.0 \\
\hline 26 & Customer Point 21 & -37.76 & -33.33 & 2.5 & 0.83 & 5.5 & 7.5 & 12.0 & 14.0 \\
\hline 27 & Customer Point 22 & 23.77 & 29.08 & 2.1 & 0.70 & 5.5 & 7.5 & 16.5 & 18.5 \\
\hline 28 & Customer Point 23 & -43.03 & 20.45 & 1.4 & 0.47 & 5.5 & 7.5 & 16.5 & 18.5 \\
\hline 29 & Customer Point 24 & -35.30 & -24.90 & 1.9 & 0.63 & 4.5 & 6.5 & 16.5 & 18.5 \\
\hline 30 & Customer Point 25 & -54.76 & 14.37 & 1.4 & 0.47 & 6.5 & 8.5 & 13.5 & 15.5 \\
\hline 31 & Customer Point 26 & -49.33 & 33.37 & 0.6 & 0.20 & 4.5 & 6.5 & 12.0 & 14.0 \\
\hline 32 & Customer Point 27 & 57.40 & 23.82 & 1.6 & 0.53 & 5.5 & 7.5 & 16.5 & 18.5 \\
\hline 33 & Customer Point 28 & -22.75 & 55.41 & 0.9 & 0.30 & 5.5 & 7.5 & 13.5 & 15.5 \\
\hline 34 & Customer Point 29 & -56.62 & 73.34 & 2.0 & 0.67 & 5.5 & 7.5 & 16.5 & 18.5 \\
\hline 35 & Customer Point 30 & -38.56 & -3.70 & 1.3 & 0.43 & 4.5 & 6.5 & 12.0 & 14.0 \\
\hline 36 & Customer Point 31 & -16.78 & 19.54 & 1.0 & 0.33 & 5.5 & 7.5 & 15.5 & 17.5 \\
\hline 37 & Customer Point 32 & -11.56 & 11.62 & 1.6 & 0.53 & 4.5 & 6.5 & 10.5 & 12.5 \\
\hline 38 & Customer Point 33 & -46.55 & 97.97 & 1.9 & 0.63 & 4.5 & 6.5 & 9.5 & 11.5 \\
\hline 39 & Customer Point 34 & 16.23 & 9.32 & 2.2 & 0.73 & 5.5 & 7.5 & 12.0 & 14.0 \\
\hline 40 & Customer Point 35 & 1.29 & 7.35 & 1.4 & 0.47 & 5.5 & 7.5 & 16.5 & 18.5 \\
\hline 41 & Customer Point 36 & -26.40 & 29.53 & 1.0 & 0.33 & 6.5 & 8.5 & 14.0 & 16.0 \\
\hline 42 & Customer Point 37 & 4.35 & 14.69 & 1.1 & 0.37 & 5.5 & 7.5 & 15.5 & 17.5 \\
\hline 43 & Customer Point 38 & -50.67 & -23.13 & 1.5 & 0.50 & 5.5 & 7.5 & 16.5 & 18.5 \\
\hline 44 & Customer Point 39 & -22.83 & -9.81 & 1.3 & 0.43 & 4.5 & 6.5 & 12.0 & 14.0 \\
\hline 45 & Customer Point 40 & -71.10 & -18.62 & 1.5 & 0.50 & 5.5 & 7.5 & 16.5 & 18.5 \\
\hline 46 & Customer Point 41 & -7.85 & 32.07 & 0.8 & 0.27 & 5.5 & 7.5 & 16.5 & 18.5 \\
\hline 47 & Customer Point 42 & 11.88 & -24.93 & 2.2 & 0.73 & 5.5 & 7.5 & 11.5 & 13.5 \\
\hline 48 & Customer Point 43 & -18.93 & -23.73 & 2.4 & 0.80 & 5.5 & 7.5 & 16.5 & 18.5 \\
\hline 49 & Customer Point 44 & -11.92 & 11.76 & 0.3 & 0.10 & 5.5 & 7.5 & 16.5 & 18.5 \\
\hline 50 & Customer Point 45 & 29.84 & 11.63 & 2.5 & 0.83 & 5.5 & 7.5 & 11.5 & 13.5 \\
\hline 51 & Customer Point 46 & 12.27 & -55.81 & 1.9 & 0.63 & 4.5 & 6.5 & 13.5 & 15.5 \\
\hline 52 & Customer Point 47 & -37.93 & -21.61 & 2.1 & 0.70 & 5.5 & 7.5 & 12.0 & 14.0 \\
\hline 53 & Customer Point 48 & 42.88 & -2.97 & 1.0 & 0.33 & 5.5 & 7.5 & 16.5 & 18.5 \\
\hline
\end{tabular}


Table 2. Optimal distribution path of each vehicle in the traditional mode

\begin{tabular}{c|c|c}
\hline Vehicle & Customers & Route and arrival time \\
\hline 1 & 6 & C-20-33-13-8-5-29-C \\
\hline 2 & 8 & $6-6.53-7.36-8.49-9.21-10.03-10.63-11.83$ \\
\hline 3 & 11 & C-15-17-18-25-23-26-1-16-C \\
\hline 4 & 6 & $6-6.76-7.64-8.26-9.15-9.84-10.55-11.36-12.05-12.97$ \\
\hline 5 & 6 & C-19-41-4-14-28-36-32-44-39-31-2-C \\
\hline 6 & 5 & $6-6.14-7.17-8.02-8.66-8.97-9.87-11.27$ \\
\hline 7 & 6 & A-46-35-9-43-24-37-A \\
\hline & & $6-16-8.86-9.5-10.37-11.44-13-13.39$ \\
\hline
\end{tabular}

Table 3. Optimal distribution path of each vehicle in the proposed mode

\begin{tabular}{c|c|c}
\hline Vehicle & Customers & Route and arrival time \\
\hline 1 & 12 & A-46-42-48-11-34-10-B-45-6-3-27-22-37-A \\
& & $6-7.16-8.3-9.66-10.32-10.71-11.56-12.26-12.43-13.59-13.81-14.66-15.76-16.86-17.25$ \\
\hline 2 & 13 & C-33-20-1-14-28-32-41-4-19-B-31-44-35-43-D \\
\hline 3 & 13 & $6-6.83-7.72-8.62-9.2-9.55-10.6-11.48-12.38-13.25-14.68-15.32-15.8-16.13-17.22-18.47$ \\
\hline 4 & 5 & B-7-9-39-24-47-30-D-21-12-38-40-17-23-2-D \\
\hline \multirow{2}{*}{5} & 5 & D-6.28-7.14-7.91-8.67-9.37-10.37-10.94-11.51-12.48-12.82-13.67-15.01-16-16.79-16.75 \\
\hline & & $6-6.36-6.94-7.78-8.31-9.32-10.01$ \\
\hline
\end{tabular}

\section{Comparative analysis}

Table 4 compares the optimal distribution plans of the two distribution modes. Under the traditional mode, the total travel distance was $1,393.45 \mathrm{~km}$, and the total distribution cost was RMB 25,920.97 yuan, including RMB 577.36 yuan of carbon emissions cost. Under the proposed mode, the total travel distance was $1,337.27 \mathrm{~km}$, and the total distribution cost was RMB 23,699.18 yuan, including RMB 501.73 yuan of carbon emissions cost.

As shown in Figure 4, the consumers in the traditional mode were served by region, and each vehicle must return to the original DC after completing the tasks in the responsible region. As shown in Figure 5, the proposed mode realized transregional distribution: after completing the current task, each vehicle can stopover or replenish its cargoes at a nearby DC; then, the vehicle can continue with distribution until completing all consumer orders in the whole network. Compared with the traditional mode, the proposed mode can distribute fresh food efficiently with a few vehicles, a 
short distance and a low cost, and saves resources in the return trips of vehicles. Besides, the proposed mode can effectively reduce the carbon emissions cost, which is in line with the green development of the enterprise.

Table 4. Distribution index comparison between the two distribution modes

\begin{tabular}{c|c|c|c|c|c|c|c}
\hline $\begin{array}{c}\text { Distribution } \\
\text { mode }\end{array}$ & $\begin{array}{c}\text { Distribution } \\
\text { distance }\end{array}$ & $\begin{array}{c}\text { Total } \\
\text { distribution } \\
\text { cost }\end{array}$ & $\begin{array}{c}\text { Dispatch } \\
\text { cost }\end{array}$ & $\begin{array}{c}\text { Transportation } \\
\text { cost }\end{array}$ & $\begin{array}{c}\text { Time } \\
\text { penalty } \\
\text { cost }\end{array}$ & $\begin{array}{c}\text { Cost of } \\
\text { goods } \\
\text { damage }\end{array}$ & $\begin{array}{c}\text { Cost of } \\
\text { carbon } \\
\text { emissions }\end{array}$ \\
\hline $\begin{array}{c}\text { District } \\
\text { distribution }\end{array}$ & 1393.45 & 25920.97 & 4200.0 & 13934.50 & 257.50 & 6951.61 & 577.36 \\
\hline $\begin{array}{c}\text { Joint } \\
\text { distribution }\end{array}$ & 1337.27 & 23699.18 & 3000.0 & 13372.70 & 253.50 & 6571.25 & 501.73 \\
\hline
\end{tabular}

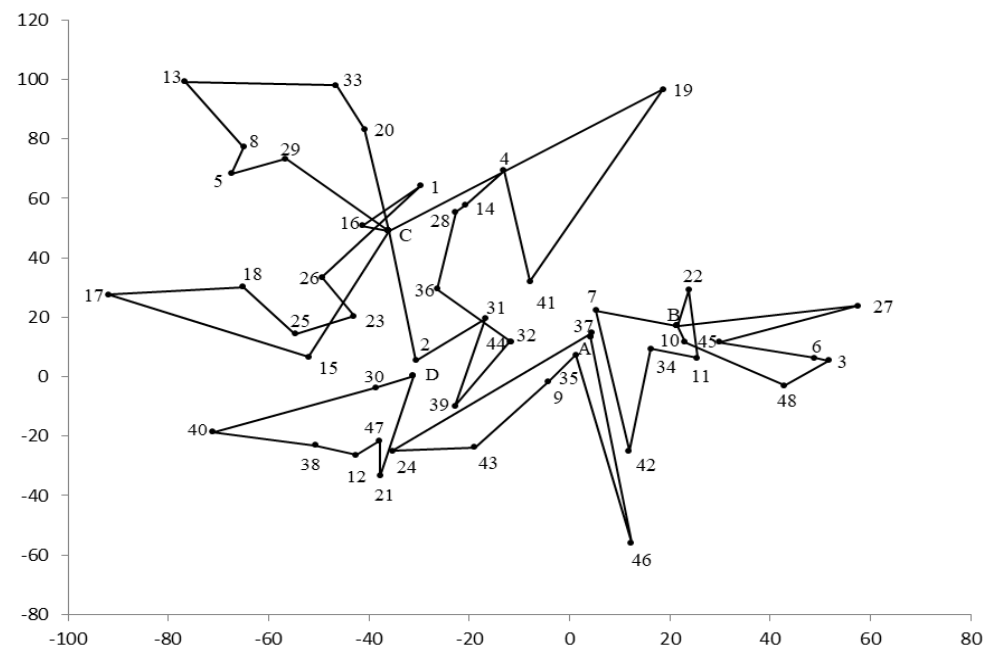

Figure 4. The optimal path for the traditional mode

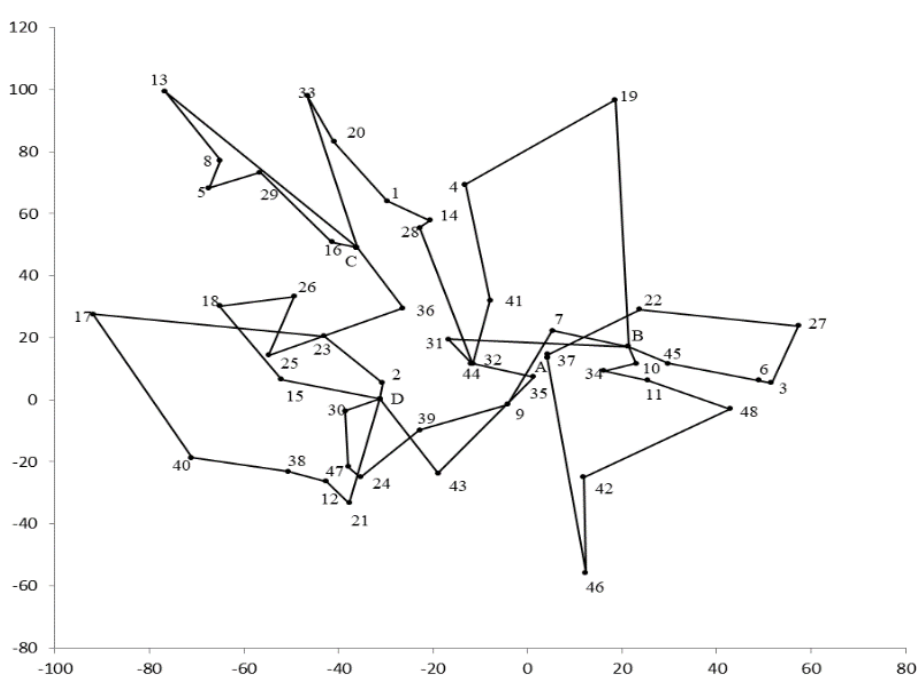

Figure 5. The optimal path for the proposed mode 


\section{Conclusions}

Targeting the fresh food distribution, this paper puts forward a semi-open multi-DC CCD model based on multi-DC resource sharing and time window constraint. The proposed model was solved by the AFSA-ACA hybrid algorithm. The semi-open multiDC distribution mode was proved to have smaller total travel distance and total cost than the traditional regional distribution mode, and, in particular, a much lower carbon emissions cost. The semi-open multi-DC distribution mode provides enterprises an important tool to save resources, realize multi-DC resource sharing, maximize economic and environmental benefits. The research findings lay a solid theoretical basis for the distribution of fresh food.

Although the content of this paper provides some reference value for fresh product distribution enterprises in planning low-carbon distribution routes, the model built in this paper is based on certain assumptions and constraints. So the research is still incomplete. This paper assumes that the same type of vehicle, the same temperature and uniform speed are constraints for distribution. Multi-type vehicle, multi-temperature joint distribution and the variable speed can be considered in the study of path optimization CCD with multi-DC considering carbon emissions in future researches.

Acknowledgements. The research of this paper is supported by Social Science Foundation of Hebei Province (HB17GL022), Research Projects of Innovative Talents Training Fund of Hebei Province (A2016001120), Project of Scientific Research Project of Hebei Provincial Education Department (SD181012, SD161009).

\section{REFERENCES}

[1] Adelzadeh, M., Asl, V. M., Koosha, M. (2014): A mathematical model and a solving procedure for multi-depot vehicle routing problem with fuzzy time window and heterogeneous vehicle. - The International Journal of Advanced Manufacturing Technology 75(5-8): 793-802.

[2] Baldacci, R., Mingozzi, A., Roberti, R., Calvo, R. W. (2013): An exact algorithm for the two-echelon capacitated vehicle routing problem. - Operations Research 61(2): 298-314.

[3] Bao, C. L., Zhang, S. B. (2018): Route optimization of cold chain logistics in joint distribution: with consideration of carbon emission. - Industrial Engineering and Management 23(5): 95-100, 107.

[4] Chaabane, A., Ramudhin, A., Paquet, M. (2012): Design of sustainable supply chains under the emission trading scheme. - International Journal of Production Economics 135(1): 37-49.

[5] Dai, X. J., Liang, C. J. (2017): Cold storage type of multi-temperature distribution problem with the soft time windows. - Journal of Chongqing Normal University (Natural Science Edition) 34(5): 18-25.

[6] Dantzig, G. B., Ramser, J. H. (1959): The truck dispatching problem. - Management Science 6(1): 80-91.

[7] Dorigo, M., Gambardella, L. M. (1997): Ant colony system: a cooperative learning approach to the traveling salesman problem. - IEEE Transactions on Evolutionary Computation 1(1): 53-56.

[8] Duro, J. (2013): International mobility in carbon dioxide emissions. - Energy Policy 55: 208-216. 
[9] Fan, H. M., Yang, X., Li, D., L. Y., Liu, P. C., Wu, J. X. (2018): Half-open multi-depot vehicle routing problem based on joint distribution mode of fresh food. - Computer Integrated Manufacturing System 25(1): 1-15.

[10] Fan, L. N., Dong, D. Y., Li, J. Y., Liu, C., Ding, Y. (2017): Route optimization of cold chain logistics based on fresh agricultural products. - Journal of Shenyang University (Natural Science Edition) 29(2): 125-131.

[11] Fan, S. Q., Lou, D., Sun, Y. (2017): Optimization study on vehicle distribution routing of cold-chain logistics for fresh agricultural products. - Storage and Process 17(6): 106-111.

[12] Gac, A. (1996): The cold chain and the greenhouse effect. - Comptes Rendus de l'Academie d'Agriculture de France 82(6): 119-133.

[13] Ge, X. L., Xu, M. Z., Wang, W. X. (2016): Route optimization of urban logistics in joint distribution. - Control and Decision-Making 31(3): 503-512.

[14] Ho, W., Ho, G. T. S., Ji, P., Lau, H. (2007): A hybrid genetic algorithm for the multidepot vehicle routing problem. - Engineering Applications of Artificial Intelligence 21(4): 548-557.

[15] James, S. J., James, C., Sant'Ana, A. D. S. (2010): The food cold-chain and climate change. - Food Research International 43(7): 1944-1956.

[16] Kang, K., Han, J., Pu, W., Ma, Y. F. (2019): Optimization research on cold chain distribution routes considering carbon emissions for fresh agricultural products. Computer Engineering and Application 55(2): 259-265.

[17] Lan, H. J., Xue, H. L., Tian, Y. B. (2013): Classification and application on food cold chain collaborative distribution models. - LISS 2012: 123-128.

[18] Li, X. L., Shao, Z. J., Qian, J. X. (2002): An optimizing method based on autonomous animals fish-swarm algorithm. - System Engineering Theory and Practice 22(11): 32-38.

[19] Liang, C. J., Huang, T., Xu, D. H., Ding, Y. (2016): A solution for cold chain distribution with fuzzy time window based on improved genetic algorithm. - Journal of Guangxi University (Natural Science Edition) 41(3): 826-835.

[20] Liu, C. Y. (2013): An improved adaptive genetic algorithm for the multi-depot vehicle routing problem with time window. - Journal of Networks 8(5): 1035.

[21] Liu, J. L., Guo, X. P. (2016): Multi-depot open vehicle routing problem with exclusive products and heterogeneous vehicles. - Journal of Systems Management 25(1): 129-138.

[22] Liu, R., Jiang, Z., Fung, R. Y. K., Chen, F., Liu, X. (2010): Two-phase heuristic algorithms for full truckloads multi-depot capacitated vehicle routing problem in carrier collaboration. - Computers \& Operations Research 37(5): 950-959.

[23] Palmer, A. (2007): The development of an integrated routing and carbon dioxide emissions model for goods vehicles. - PhD Thesis, School of Management, Cranfield University.

[24] Vanek, F., Sun, Y. (2008): Transportation versus perishability in life cycle energy consumption: a case study of the temperature-controlled food product supply chain. Transportation Research Part D Transport \& Environment 13(6): 383-391.

[25] Wang, S. Y., Sun, H., Mou, J. J. (2018): Optimization of cold-storage multi-temperature joint distribution based on stochastic demands. - Systems Engineering-Theory Methodology Application 27(4): 712-721.

[26] Wang, Y., Zhang, J., Liu, Y., Xu, M. Z. (2019): Optimization method study of fresh good logistics distribution based on time window and temperature control. - Control and Decision-Making. DOI: 10.13195/j.kzyjc.2018.1662.

[27] Xiao, Y., Zhao, Q., Kaku, I, Xu, Y. C. (2012): Development of a fuel consumption optimization model for the capacitated vehicle routing problem. - Computers \& Operations Research 39(7): 1419-1431.

[28] Yang, X., Fan, H. M., Zhang, X. N., Li, Y. (2016): Optimization of multi-deport open vehicle routing problem with fuzzy time window. - Computer Integrated Manufacturing System 22(7): 1768-1778. 
[29] Yu, B., Xie, Y. X. (2011): A parallel improved ant colony optimization for multi-depot vehicle routing problem. - The Journal of the Operational Research Society 62(1): 183188. 